\title{
The Treatment of Acne with the Addition and Subtraction of Loquat Qingfei Yin: A Meta-Analysis
}

\author{
Wenjun Xue ${ }^{1 *}$, Pengguang $\mathrm{Xu}^{2}$ \\ ${ }^{1}$ Shannxi University of Traditional Chinese Medicine, Xianyang 712046, Shannxi Province, China \\ ${ }^{2}$ Affiliated Hospital of Shannxi University of Traditional Chinese Medicine, Xianyang 712046, Shannxi Province, China \\ *Corresponding author: Wenjun Xue, sxxa_0@163.com
}

\begin{abstract}
Objective: To systematically evaluate the efficacy and safety of Loquat Qingfei Yin (LQFY) in the treatment of acne. Methods: Using computer retrieval, comprehensive collection of the full text database of Chinese journals till December 2020 (CNKI), Wanfang Data Knowledge Service Platform (Wanfang), Viper Database (VIP), PubMed, Cochrane Library, Medline were included in the LQFY addition or subtraction treatment of acne randomized controlled trial (RCT). Screening of the literatures included in the Note Express 3.2.0. Assessment of the risk of bias in trials using Cochrane collaboration tools, and used Rev Man 5.3 to evaluate the curative effect. Results: 25 RCT, all in Chinese, there were 2257 cases. Of the 1216 cases who were treated, control group was 1041 cases. A random effect model was used for Meta analysis. The results showed that the effective rate of the treatment group was significantly higher than that of the control group, Differences were statistically significant $(\mathrm{P}=0.94, \mathrm{I} 2=0 \%), \mathrm{RR}=2.87$, CI [2.25,95 per cent 3.67], $\mathrm{P}<0.00001$. Conclusion: There are limited evidence that LQFY is safe and effective in treating acne. However, this conclusion needs to be confirmed by a more largescale, multi-center, high-quality RCTs.
\end{abstract}

Keywords: LQFY addition or subtraction; Acne; Randomized controlled trial (RCT); Meta analysis

Publication date: July 2021; Online publication: July 31, 2021

\section{Introduction}

Acne, is a chronic inflammatory skin disease of the hair follicle sebaceous glands. Epidemiological studies show that $80 \%-90 \%$ of adolescents have experienced acne ${ }^{[1]}$. In Chinese medicine, they call it "blisters," which are considered to be excessive in the Yang heat of the lung, or addicted to eating fat, sweet and spicy. This causes the gastrointestinal damp-heat to get mixed, thus leads to release of heat on top of the spleen, making the stomach weak. The damp-heat is contained, the stagnation and heat are retained, the liquid turns into phlegm, and the damp-heat phlegm and blood stasis are trapped in the skin. LQFY is an ancient acne treatment that clears "lung qi" (TCM term for heatiness), clears heat and diarrhea heat, detoxifies, and body dampness.

In order to explore the effectiveness and safety of LQFY in the treatment of acne, this study applied evidence-based medicine principles and methods to comprehensively search and collect RCT of LQFY in the treatment of acne. The effectiveness and safety of the treatment of acne were evaluated to provide a more reliable basis for the treatment of acne in the future.

\section{Clinical Data and Methods}

\subsection{Literature search}

Searched six electronic databases for RCT of acne treatment with LQFY, which was included in the 
database until December 25, 2020. Search libraries include: CNKI, Wanfang, VIP Database, PubMed, Library of Congress, Wiley Online Library. The Chinese database uses "Pi-pa Qingfei Yin," "LQFY" and "acne" as search terms. In addition, it adopts the subject/ keyword/ full text search method according to the characteristics of each database. The English database uses "Pi-Pa Qing Fei Granule," "LQFY" and "acne" as search terms. Finally, a total of $25^{[2-26]}$ RCT were included. Meta-analysis was used to evaluate the clinical efficacy and adverse reactions of LQFY in the treatment of acne.

\subsection{Document Acceptance Standard}

\subsubsection{Inclusion criteria}

(1) This is a RCT type of the literature, published at home and abroad, and the number of samples included in the literature are more than 10.

(2) All patients in the study should meet the diagnostic criteria, and the level of specific diagnostic criteria is not limited. The age and race of the patients are not limited.

(3) The intervention in the treatment group is LQFY (addition and subtraction), and the control group adopts other treatment methods.

(4) There are clear curative effect evaluation standards.

(5) The data is completed.

\subsubsection{Exclusion standards}

(1) Non-randomized controlled test

(2) Repeated publications

\subsubsection{Outcome indicators}

Clinical efficacy, adverse reactions, recurrence and follow-up.

\subsection{Literature screening and data extraction}

The articles screened by two people. When there were objections, the third person joined the discussion. The data extraction table mainly includes the basic information of the research (author name, research title, publication year, country/region), research characteristics (sample size, case source, age, diagnostic criteria, inclusion criteria and exclusion criteria), literature quality evaluation indicators (random plan generation, hidden assignment, blinding, incomplete result data, selective reporting, other deviations, loss to follow-up) and measurement data of outcome indicators. The criteria's used for this study are clinical efficacy, adverse reactions, time to regression of skin lesions, and recurrence.

\subsection{Literature quality evaluation}

The Cochrane collaborative tool was used to evaluate the methodological quality of each included trial. The criteria for assessing the risk of bias in literature quality includes the random sequence generation methods, allocation concealment, blinding, incomplete result data, selective reporting, and other seven aspects biases.

\subsection{Statistical analysis}

Rev Man 5.3 software was used for meta-analysis, which included the literature. Binary variables use relative risk (RR) and $95 \%$ confidence interval $(95 \% \mathrm{CI})$ as the efficacy statistics, to determine the heterogeneity of the trial based on the results of $\mathrm{I}^{2}$ test. When $\mathrm{I}^{2}<50 \%$, it indicates the heterogeneity between the lower trials, where the fixed-effects model was used. In addition, when $\mathrm{I}^{2}>50 \%$, the random-effects model was used. If the result of the heterogeneity test is $\mathrm{P} \leq 0.05$ and $\mathrm{I}^{2} \geq 50 \%$, the random effects model will be used for the combined analysis of the efficacy. Therefore, the factors that may cause heterogeneity will be analyzed by sub-groups. Potential publication bias was analyzed using "inverted funnel chart." 


\section{Results}

\subsection{Search results}

According to the search strategy, none of the English databases retrieved articles that fit this study. The Chinese database retrieved a total of 972 articles. After deleting duplicate articles, 387 articles remained. According to the criteria for inclusion and ranking, 66 articles were deleted after reading the title and abstract. After the full text, $25^{[2-26]}$ RCT were finally included as shown in Table 1.

Table 1. Basic characteristics of included studies

\begin{tabular}{|c|c|c|c|c|}
\hline $\begin{array}{l}\text { Included } \\
\text { studies }\end{array}$ & $\begin{array}{c}\text { Sample } \\
\text { size } \\
(\mathrm{T} / \mathrm{C})\end{array}$ & Block method & $\begin{array}{l}\text { Intervention } \\
\text { measure }\end{array}$ & Course \\
\hline Wang Kui 2019 & $50 / 50$ & $\begin{array}{l}\text { Random order } \\
\text { random }\end{array}$ & Per + Via & $4 w$ \\
\hline $\begin{array}{l}\text { Zhang Rui } \\
2019\end{array}$ & $40 / 40$ & $\begin{array}{c}\text { Table of random } \\
\text { number }\end{array}$ & Dst & $4 w$ \\
\hline Zheng Di 2019 & $50 / 50$ & $\begin{array}{c}\text { Table of random } \\
\text { number }\end{array}$ & Mino & $8 w$ \\
\hline $\begin{array}{l}\text { Niu Chunyan } \\
2016\end{array}$ & $28 / 28$ & Visit order & Mino + Tret & $4 w$ \\
\hline Hu Yan 2016 & $30 / 30$ & Random & Tetr + Vit B6 & Not mentioned \\
\hline $\begin{array}{l}\text { Lee Zongchao } \\
2016\end{array}$ & $48 / 46$ & $\begin{array}{c}\text { Table of random } \\
\text { number }\end{array}$ & $\mathrm{Xcw}$ & $8 w$ \\
\hline $\begin{array}{l}\text { Wang Xuejun } \\
2014\end{array}$ & $35 / 35$ & Random & $\mathrm{Xcw}$ & $4 w$ \\
\hline $\begin{array}{l}\text { Xu Guangcang } \\
2013\end{array}$ & $102 / 78$ & Random & Dst & $4 w$ \\
\hline $\begin{array}{l}\text { Wang Shuai } \\
2013\end{array}$ & $61 / 60$ & Random & Mino & $12 w$ \\
\hline $\begin{array}{l}\text { Ma Tianlong } \\
2013\end{array}$ & $42 / 42$ & Random & $\begin{array}{c}\text { Vit } C+\text { Roxi }+ \text { Vit } \\
\text { B6 }\end{array}$ & $\begin{array}{l}\text { Therapy group } 3 w \text {, } \\
\text { Control group } 4 w\end{array}$ \\
\hline $\begin{array}{l}\text { Huang } \\
\text { Shancong } 2013\end{array}$ & $35 / 35$ & Random & Yq & $8 w$ \\
\hline $\begin{array}{l}\text { Chen Liangjin } \\
2011\end{array}$ & $53 / 47$ & $\begin{array}{l}\text { Random time } \\
\text { random }\end{array}$ & Via + Per & $4 w$ \\
\hline $\begin{array}{l}\text { Zhang Yuan } \\
2011\end{array}$ & $85 / 50$ & Random & Via & $4 w$ \\
\hline
\end{tabular}


Chen Zhongwei

2010

Jiang Zhengbin

2010

Xi Jianning

2009

Qi Haiwen

2009

Liang Xisen

2009

Shi Xuebo

2008

Zhang Lingling

2006

$60 / 60$

Wang Shaokun

2006

Fu Peijun 2005

ShenJie 2003

Dianwen 2003

$40 / 40$

$60 / 48$

Ji Hongjun
Random

Random

$\mathrm{Ccl}$

$6 w$

Table of random

number

Roxi + vit B6 + Zs

$8 w$

Random

Xcw

$8 w$

Random

Ery $+\mathrm{ZS}+5 \%$

Sulfur Frost

30d

$0.1 \%$ Vit $\mathrm{A}$ acid

cream $(+1 \%$

Clindamycin Gel)

Table of random

number

Via

4w

Vim E

$3 w$

Random

Random

$\mathrm{Sr}$

$12 \mathrm{w}$

60/40 Random

Clin + MB2

4w

Tetr + vit B6

$4 w$

2002
Random

Random
Tetr + Sim

$4 w$

Peroxybenzoyl Gel (Per); Viaminate Capsules (Via); Danshentong Capsules (Dst); Minocycline (Mino); Tretinoincream (Tret); Tetracycline Tablets (Tetr); Xiao cuo wan (Xcw); Roxithromycin tablets (Roxi); Yiqing Capsule (Yq); Qing re an chuang wan (Qrac); Cuo Chuang Ling Granules (Ccl); Zinc Sulfate (Zs); Erythromycin (Ery); Sanrui capsule (Sr); Clindamycin (Clin); Multivitamin B2 Tablets (MB2); Simitidine tablets (Sim); Vitamin (Vit).

\subsection{Bias risk results}

A total of five articles ${ }^{[11-13,15-16]}$ articles are grouped according to the random number table, and one ${ }^{[5]}$ article was based on the order of visits for grouping. One article ${ }^{[2]}$ was grouped randomly according to the order of visits, and another ${ }^{[13]}$ article was grouped randomly according to the time of visits, 17 articles [6,8-12,14-16,18-20,22-26] were grouped by random method. All the literature did not describe allocation concealment and blinding, and its risk of bias was judged to be unclear. In all the literature, there was no case of dropout, thus no intention-to-treat analysis was used, as the risk of bias was low. A funnel chart with the RR value of $25^{[2-26]}$ articles as the $y$-axis and $S E(\log [R R])$ as the $x$-axis was derived. The distribution chart shows that the article samples are roughly distributed around the overall effect, arranged 
symmetrically around the centerline, and a small part of it was tilted. Therefore, it indicated that the bias of the included literatures in this study was relatively small. Figure 1. shows the methodological quality assessment results of all the included studies. Figure 2. shows the percentage results of the risk of bias for each of the included trials, and Figure 3. shows the clinical funnel.

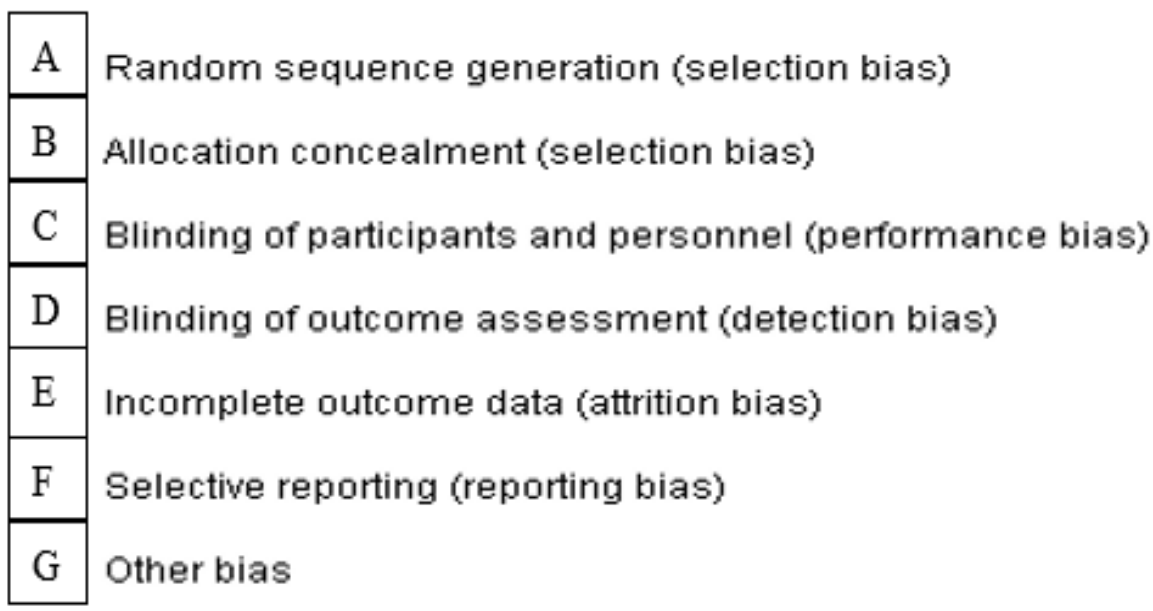

\begin{tabular}{|c|c|c|c|c|c|c|c|}
\hline \multirow[b]{2}{*}{ Chen Liangiin2011 } & A & $\mathrm{B}$ & $\mathrm{C}$ & $\mathrm{D}$ & $\mathrm{E}$ & $\mathrm{F}$ & $\mathrm{G}$ \\
\hline & $\rightarrow$ & $?$ & $?$ & & $\rightarrow$ & $\rightarrow$ & \\
\hline \multirow{2}{*}{$\begin{array}{r}\text { Chen Zhongweizo10 } \\
\text { Fu Peijunzo0s }\end{array}$} & $\rightarrow$ & $\geq$ & $\geq$ & $\div$ & $\leftarrow$ & $\leftarrow$ & \\
\hline & $\rightarrow$ & $\geq$ & $\geq$ & $\leftarrow$ & $\leftarrow$ & $\leftarrow$ & G \\
\hline \multirow{3}{*}{$\begin{array}{r}\text { Huang Shancong } 2013 \\
\text { Hu Yan2016 } \\
\text { Jiang Zhengbinz010 }\end{array}$} & $\rightarrow$ & $?$ & 3 & 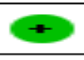 & $\div$ & $\leftarrow$ & - \\
\hline & 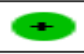 & $?$ & $?$ & + & 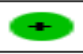 & + & \\
\hline & $\rightarrow$ & $?$ & 3 & 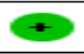 & $\rightarrow$ & 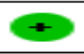 & $\leftarrow$ \\
\hline $\begin{array}{l}\text { Jiang Zhengbinzo10 } \\
\text { Ji Hongiunzo02 }\end{array}$ & $\rightarrow$ & 3 & $\geq$ & $\leftarrow$ & $\rightarrow$ & $\leftarrow$ & $\leftarrow$ \\
\hline Liang xisenzoog & 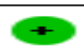 & $\geq$ & $\geq$ & + & $\leftarrow$ & 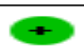 & \\
\hline Li Dianwenzo03 & $\rightarrow$ & $?$ & $?$ & 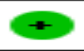 & $\rightarrow$ & 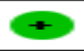 & \\
\hline \multirow{2}{*}{$\begin{array}{l}\text { Li Zongchaoz016 } \\
\text { Ma Tianlong } 2013\end{array}$} & $\rightarrow$ & $?$ & $?$ & $\leftarrow$ & $\leftarrow$ & $\leftarrow$ & $\leftarrow$ \\
\hline & 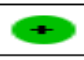 & $\geq$ & 3 & $\leftarrow$ & $\leftarrow$ & 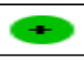 & - \\
\hline \multirow{2}{*}{$\begin{array}{r}\text { Niu Chunyan2015 } \\
\text { Qi Haiwen2009 }\end{array}$} & & $?$ & 3 & 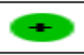 & + & + & 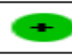 \\
\hline & 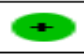 & $?$ & $?$ & 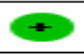 & $\leftarrow$ & $\leftarrow$ & $\leftarrow$ \\
\hline Shen Jiezons & $\rightarrow$ & $\geq$ & $\geq$ & $\leftarrow$ & $\leftarrow$ & $\leftarrow$ & 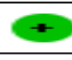 \\
\hline Shi xuebozoos & $\leftrightarrow$ & $?$ & 3 & $\leftarrow$ & $\leftarrow$ & $\leftarrow$ & \\
\hline \multirow{2}{*}{$\begin{array}{r}\text { Wang kui } 2019 \\
\text { wang Shaokunzo0s }\end{array}$} & $\rightarrow$ & $?$ & 3 & $\rightarrow$ & $\div$ & $\rightarrow$ & $\div$ \\
\hline & 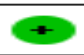 & 3 & 3 & + & $\leftarrow$ & $\leftarrow$ & $\leftarrow$ \\
\hline $\begin{array}{r}\text { Wang Shaokun2006 } \\
\text { wang Shuai2013 }\end{array}$ & 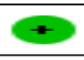 & $\geq$ & 3 & $\leftarrow$ & $\div$ & 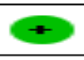 & $\div$ \\
\hline \multirow{2}{*}{$\begin{array}{r}\text { Wang Xuejun } 2014 \\
\text { xi Yanning } 2009\end{array}$} & $\rightarrow$ & $\geq$ & $\geq$ & $\rightarrow$ & $\rightarrow$ & $\rightarrow$ & $\leftarrow$ \\
\hline & $\Leftrightarrow$ & $?$ & $?$ & $\leftarrow$ & $\leftarrow$ & $\leftarrow$ & $\leftarrow$ \\
\hline \multirow{2}{*}{$\begin{array}{l}\text { xu Guangcang } 2013 \\
\text { Zhang Lingling } 2006\end{array}$} & $\Leftrightarrow$ & $?$ & $?$ & $\leftarrow$ & $\leftarrow$ & $\rightarrow$ & $\leftarrow$ \\
\hline & $\rightarrow$ & $?$ & $?$ & $\rightarrow$ & $\leftarrow$ & $\rightarrow$ & 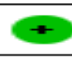 \\
\hline \multirow{2}{*}{$\begin{array}{l}\text { Znang Ruizo19 } \\
\text { Zhang Yuanz011 }\end{array}$} & 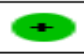 & $\geq$ & 3 & $\div$ & $\leftarrow$ & + & $\div$ \\
\hline & 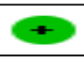 & $?$ & 3 & $\leftarrow$ & $\leftarrow$ & $\leftarrow$ & $\leftarrow$ \\
\hline $\begin{array}{r}\text { Zhang Yuanz011 } \\
\text { Zheng Di2019 }\end{array}$ & $\Leftrightarrow$ & $?$ & 3 & 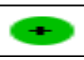 & $\leftarrow$ & $\leftarrow$ & $\div$ \\
\hline
\end{tabular}

Figure 1. Methodological quality evaluation in the study 


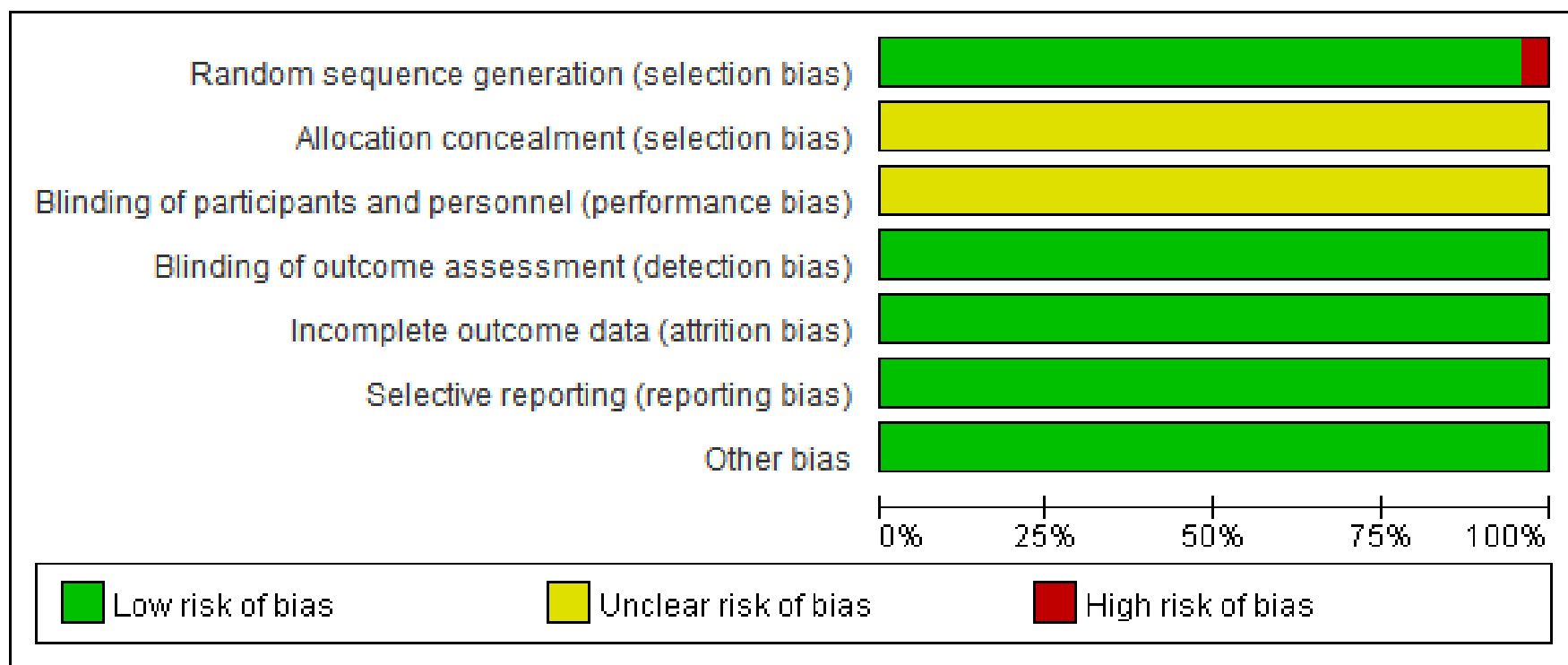

Figure 2. Percentage results of all the bias risk included in the test

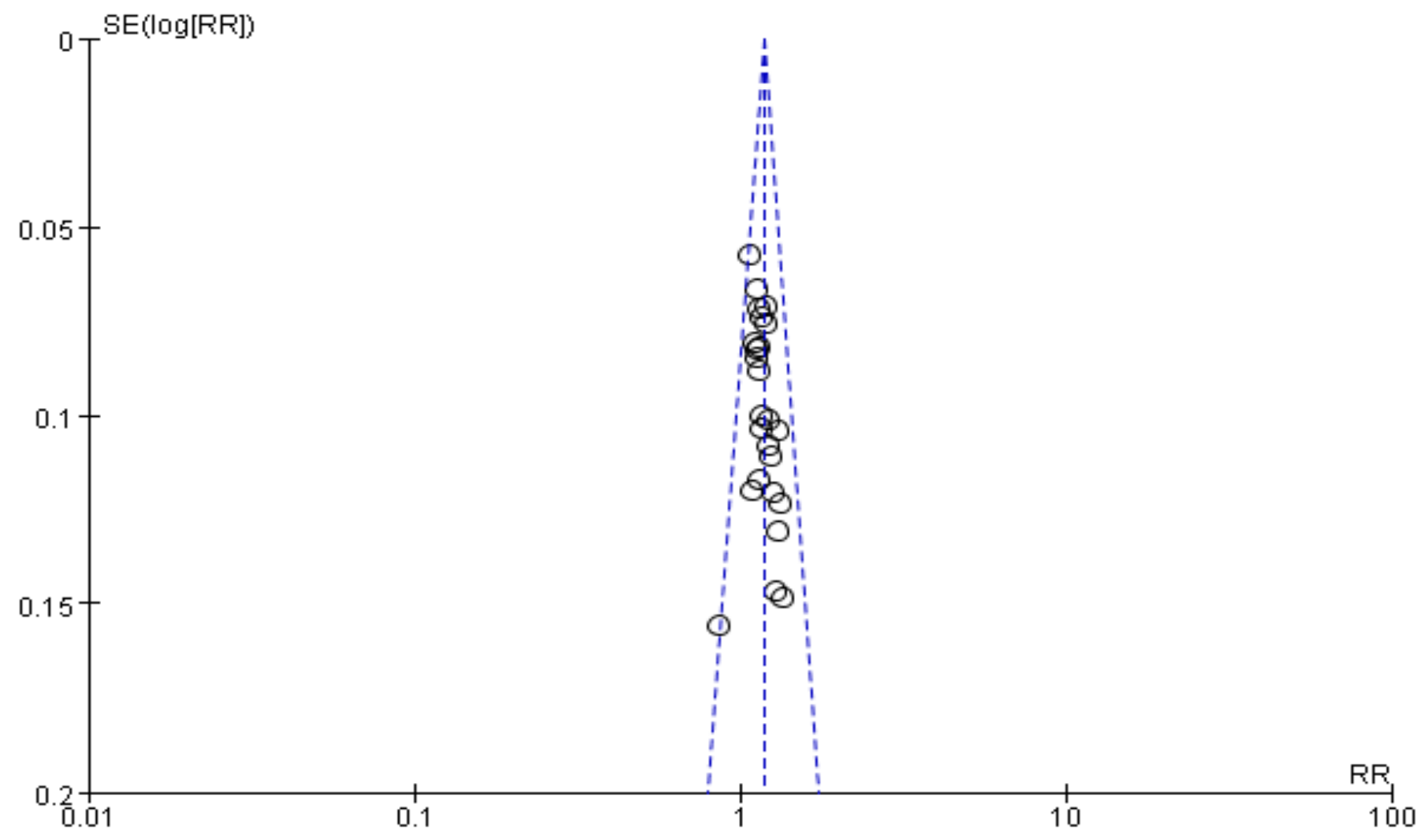

Figure 3. Clinical funnel

\subsection{Results}

\subsubsection{Meta analysis}

The 25 studies provided data on the treatment of acne, trial group of 1216 cases, control group of 1041 cases, $\mathrm{P}=0.94, \mathrm{I} 2=0 \%$, showed low heterogeneity, thus the Meta analysis results are $[\mathrm{OR}=2.87,95 \% \mathrm{CI}$ [2.25, 3.67], $\mathrm{P}<0.00001]$. Mainly, the trial and control groups, showed that the LQFY was more effective in clinical efficacy than other therapies. See Figure 4. 


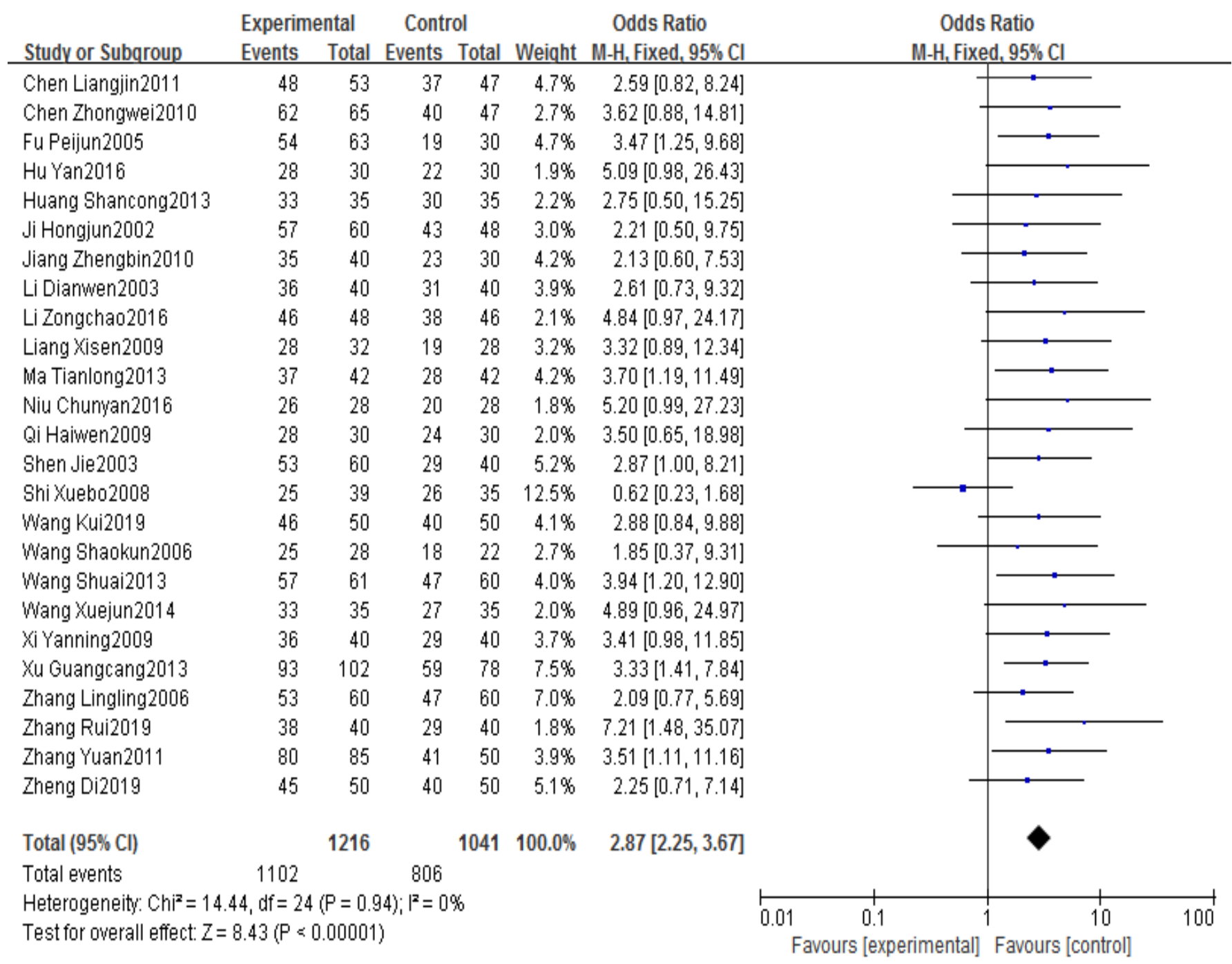

Figure 4. Meta-analysis of the clinical efficacy of LQFY in the treatment of acne

\subsubsection{Security assessment}

Among the $11^{[2-3,6,16,18-21,23,25-26]}$ articles reported adverse reactions, of which one ${ }^{[2]}$ study mentioned that the experimental group and the control group did not have adverse reactions. Moreover, three [19,21,23] studies found that the experimental group had no adverse reactions, contrarily, based on the cases among the adverse reactions observed that one patient ${ }^{[3]}$ had facial flushing, one patient ${ }^{[6]}$ had loss of appetite, and 19 patients had diarrhea ${ }^{[16,18,20,26]}$, of which one ${ }^{[16]}$ patient was mentioned in the study as the symptoms disappeared after adjusting the medicine. Another study showed that for one patient ${ }^{[18]}$, the symptoms disappeared after taking the medicine after meals. There were 13 cases of nausea, vomiting, and upper abdominal discomfort ${ }^{[3,6,16,18,20,25]}$, and one ${ }^{[26]}$ study showed that the diarrhea symptoms disappeared after stopping the drug. A test ${ }^{[18]}$ mentioned that there were no obvious abnormalities in the measurement of blood, urine, feces, liver and kidney functions of the patient before and after the test, and one ${ }^{[25]}$ test mentioned that the blood, urine, feces and liver function of the patient were measured before and after the test, and there were no obvious abnormalities. Four ${ }^{[11,20,24,26]}$ articles recorded follow-up recurrence, of which only one article ${ }^{[11]}$ reported recurrence within a year, and the follow-up time of the remaining trials ranged from three to six months. 


\section{Discussion}

\subsection{Efficacy analysis}

A total of 25 RCT were included in this study, with a total of 2247 patients. Meta-analysis results show that LQFY has an advantage in the treatment of acne compared with other therapies.

\subsection{Limitations of this study}

(1) The included study's methodological treatment was generally poor. The use of the concealment and blinding method was not specified in any of the research, which could lead to selection or implementation bias; the result measurer's blindness is also not mentioned, which could lead to measurement bias. The majority of the follow-up period was short, which may cause the experiment's results to be bias.

(2) The literature included had no sample estimation basis, which led to the decrease of the test efficiency.

(3) The choice of intervention measures: the experimental group which only chose LQFY on the addition and subtraction basis, but the standards were different. Thus, they could only see the general trend of the treatment effect.

(4) Funnel after graph analysis, found that there may be publication bias. Thus, it is necessary to strengthen the literature search, and hope that more high-quality clinical RCT could be carried out and published.

\subsection{Implications for the future}

Although traditional Chinese medicine (TCM) is gaining popularity in society, the methodological quality of the research has not yet matched internationally recognized standards. More rigorous studies are hoped to be designed, industry-recognized diagnosis and treatment standards will be adopted, and changes in the condition will be recorded as detailed and accurately as possible throughout the research process. Also, follow-up with the patients whom have withdrawn from the trial. Adverse responses, recurrences, and other undesirable results should all be recorded and reported, and the follow-up period should be appropriately extended.

Acne is a common skin disease, and the incidence rate of acne in the Chinese population is $8.1 \%$ [27]. Modern medicine treats acne based on the principles of removing oil, dissolving cutin, sterilizing, antiinflammatory and regulating hormone levels. It is divided into general treatment and systemic treatment, including external medication, systemic oral medication and phototherapy. Among them, the commonly used antibiotics for oral medicine are: isotretinoin, anti-androgen and glucocorticoids, but these drugs have different side effects. Studies have confirmed that LQFY can inhibit sebum secretion ${ }^{[28]}$, inhibit the growth of Malassezia ${ }^{[29]}$, have anti-keratosis effect, and significantly reduce the serum level of animal acne models. Testosterone content ${ }^{[30]}$, may be the mechanism of LQFY in the treatment of acne.

This study extracts the effective data from the current existing clinical RCT, conducts systematic reviews and Meta analysis, and evaluates the clinical efficacy and safety of LQFY for acne. It is ex pected to provide reference for clinical medication. The treatment of acne provides a reliable evidence-based basis.

\section{Disclosure statement}

The author declares no conflict of interest. 


\section{References}

[1] Zhao B., 2009, Chinese Clinical Dermatology, Jiangsu Phoenix Science and Technology Press, Nanjing, 1165.

[2] Wang K, Xu M, Hu W, 2019, Observation of the Clinical Effect of Loquat Qingfei Yin in the Treatment of Acne. Chinese Medical Cosmetology. 9(11): 98-101.

[3] Zhang R, Li F, Zhang M, 2019, Evaluation of the Effect and Adverse Reaction Rate of Loquat Qingfei Yin in the Treatment of Pulmonary and Stomach Damp-Heat Facial Acne, China Medical Cosmetology. 9(6): 104-107.

[4] Zheng D, Zhang H, 2019, The Clinical Efficacy of Loquat Qingfei Yin in the Treatment of Acne Vulgaris of Lung Meridian Wind-Heat Type, Clinical Medical Journal. 47(6): 606-607.

[5] Niu C, 2016, Observation on the Efficacy of the Method of Clearing The Lungs, Purging Heat, Removing Dampness and Removing Phlegm in the Treatment of Severe Acne, Primary Medical Forum. 20(23): 3267-3269

[6] Hu Y, 2016, Observation on the Clinical Effect of Modified Loquat Qingfei Yin in the Treatment of 60 Cases of Acne, Health Today. 15(7): 19, 22.

[7] Li Z, Ye W, 2016, Observation on the Efficacy of Loquat Qingfei Yin in the Treatment of Acne Vulgaris And Its Influence On Sex Hormone Levels, Chinese Journal of Traditional Chinese Medicine. 31(2): 731-733.

[8] Wang X, Zheng N, An Y, 2014, Clinical Observation on the Treatment of Acne Vulgaris Caused By Lung And Stomach Heat With Modified Loquat Qingfei Yin, Liaoning Journal of Traditional Chinese Medicine. 41(9): 1799-1801.

[9] Xu G, Kong R, 2013, Observation on the Efficacy of Loquat Qingfei Yin in the Treatment of Acne, Journal of Practical Traditional Chinese Medicine. 29(7): 529-530.

[10] Wang S, Wu B, Zhu J, 2013, Effect of Loquat Qingfei Yin on Serum Androgen Levels in Female Patients with Acne Vulgaris. Journal of Shandong University of Traditional Chinese Medicine. 37(3): 230-231.

[11] Ma Tianlong, 2013, Modified Loquat Qingfei Yin is Effective in Treating 84 Cases of Acne, Inner Mongolia Traditional Chinese Medicine. 32(23): 12-13.

[12] Huang S, Chen L, 2013, Chen Li's Clinical Observation on Treating Acne with Loquat Qingfei Yin, Journal of Liaoning University of Traditional Chinese Medicine. 15(10): 179-181.

[13] Chen L, Zhou J, 2011, Observation on the Curative Effect of 53 Cases Of Acne Vulgaris Treated With Modified Loquat Qingfei Yin, Asia-Pacific Traditional Medicine. 07(4): 48-49.

[14] Zhang Y, Huang Y, 2011, Treatment of 135 Cases Of Acne Vulgaris with Loquat Qingfei Yin, Modern Medicine and Health. 27(3): 334-335.

[15] Chen Z, 2010, Clinical Observation on 65 Cases of Acne Treated with Loquat Qingfei Yin. Chinese Medicine Herald. 16(5): 62-63.

[16] Jiang Z, 2010, Discussion on the Incidence of Acne and Clinical Observation of Loquat Qingfei Yin in the Treatment Of Acne Vulgaris, Nanjing University of Traditional Chinese Medicine. 45. 
[17] Xi Y, 2009, Modified Loquat Qingfei Yin in the Treatment of 40 Cases of Pulmonary Acne, Journal of Traditional Chinese Medicine. 22(2): pp. 37-38.

[18] Qi H, 2009, Clinical Study of Loquat Qingfei Granules in Treating Acne of Lung Meridian WindHeat Type, Heilongjiang Academy of Traditional Chinese Medicine, 50.

[19] Liang X, Wang W, 2009, 32 Cases of Acne Vulgaris Treated with Pipi Qingfei Decoction, New Journal of Traditional Chinese Medicine. 41(11): 87-87.

[20] Shi X, Zhou R, Zhao Y, 2008, Loquat Qingfei Yin in the Treatment andPprevention of Acne Vulgaris Recurrence, Chinese Journal of Dermatology and Venereology. 22(6): 375-376.

[21] Zhang L, 2006, Observation on the Therapeutic Effect of Loquat Qingfei Yin in the Treatment Of Lung-Gastric Damp-Heat Acne, Practical Clinical Medicine of Integrated Traditional Chinese and Western Medicine. 6(3): 31-32.

[22] Wang Z, 2006, Observation on the Curative Effect of 28 Cases Of Acne Treated with Loquat Qingfei Yin, Yunnan Journal of Traditional Chinese Medicine and Materia Medica. 27(6): 25-26.

[23] Fu P, Ma S, 2005, Observation on the Curative Effect of Loquat Qingfei Yin in the Treatment Of Acne, Liaoning Journal of Traditional Chinese Medicine. 32(7): 679-680.

[24] Shen J, Wu M, 2003, Modified Loquat Qingfei Yin in the Treatment of 60 Cases of Acne Vulgaris, Heihe Science and Technology. (4): 160.

[25] Li D, Cui H, 2003, Loquat Qingfei Yin for the Treatment of Moderate to Severe Acne Vulgaris, Heilongjiang Traditional Chinese Medicine (6): 30.

[26] Ji H, Shan J, 2002, Loquat Qingfei Yin in the Treatment of Acne Vulgaris, Zhejiang Journal of Integrated Traditional Chinese and Western Medicine. 12(9): 566-567.

[27] Ju Q, Fan WX, Gu J, et al., 2019, Chinese Acne Treatment Guidelines (2019 Revised Edition), Journal of Clinical Dermatology. (9): 583-588.

[28] Cai H, Huang G, Zhao Y, et al., 2012, Mechanism of Modified Loquat Qingfei Yin in the Treatment of Malassezia Folliculitis, Contemporary Medicine. 18(22): 129-130.

[29] Zhao Y, Jiang J, Tu H, et al., 2009, Modified Loquat Qingfei Yin in the Treatment of Malassezia Folliculitis Clinical Efficacy and Mechanism of Action, Chinese Journal of Integrated Traditional Chinese and Western Medicine Dermatology and Venereology. 8(01):11-13.

[30] Xu H, Chen J, Liu J, et al., 2009, Effect of Jiawei Loquat Qingfei Granules on Anti-Keratosis and Testosterone Level in Rabbit Ear Acne Model, Chinese Journal of Dermatovenereology. 23(01): 13$15+28$. 